\title{
Macro-prediction Analysis of Cold Recycled Asphalt Pavement Performance Based on Grey Model
}

\author{
Hongjun Jing ${ }^{1,2}$, Meng Gao ${ }^{1,2,}$, Lichen Song ${ }^{3}$ and Qian Liu ${ }^{1,2}$ \\ ${ }^{I}$ College of Architecture and Civil Engineering, Xi' an University of Science and Technology, Xi'an 710054, China \\ ${ }^{2}$ Road Engineering Research Center of Xi'an University of Science and Technology, Xi'an 710054, China \\ ${ }^{3}$ Beijing Urban Construction Group Co., Ltd, Beijing 100088, China
}

Received 19 July 2021; Accepted 27 September 2021

\begin{abstract}
The cold recycling of asphalt pavement has gradually become an important tool for maintenance and reconstruction of old roads because of its economic and environmental characteristics, but it's difficult to evaluate the practical application effect of cold recycling technology due to the lack of macro-level analysis of the performance of the cold recycled pavement. To comprehensively analyze the condition of road network-level cold recycled asphalt pavement (RAP) and evaluate the value of cold recycling technology promotion, in this study the performance indexes of cold recycling technology for asphalt pavement were comprehensively analyzed, the performance and change law of cold RAP were macroscopically predicted, the evaluation index (CRPI) and road network-level fuzzy prediction evaluation system of cold recycled pavement were proposed, and the pavement surface condition index(PCI), riding quality index(RQI), rutting depth index(RDI), and pavement structural strength index (PSSI) were selected as macroscopic evaluation indexes of road network-level cold recycling pavements. Combined with the improved analytic hierarchy process, the weight of the selected index was assigned, and the evaluation levels were divided into three standards: superior, medium, and poor. Finally, based on the C\# programming language, combined with grey prediction model and integral algorithm, a fuzzy prediction and evaluation system for the performance of road network-level cold RAP was developed, and the reliability of the prediction was verified based on the actual measurement data. Results show that in the network-level fuzzy prediction and evaluation system of cold RAP technology, the weights of PCI, RQI, QDI, and PSSI are assigned as 0.20, $0.07,0.20$, and 0.53 . The predicted value of the performance prediction system for cold RAP has a good correlation with the actual test value, and the relative error is small. The system can obtain the evaluation curve of each index and calculate the comprehensive performance score, and realize the prediction of the overall development trend of cold RAP performance. It shows that the system can analyze and predict the performance of cold RAP at the macro-level. Conclusions of this study provide index system and system support for the macroscopic analysis of cold RAP performance, and also provide reference for related research work.
\end{abstract}

Keywords: Road engineering, cold recycled asphalt pavement, big data, fuzzy prediction, fuzzy evaluation

\section{Introduction}

Highway construction has progressed considerably with rapid economic development in various countries. However, the pavement performance of many highways built in early years fails to satisfy contemporary transportation demands, with some even showing damage, such as rutting, cracks, and reduction in strength. If these highways are demolished to build new ones, then a large amount of waste will be generated from old highways that will result in not only environmental destruction but also remarkably increase the demand for new materials. Recycling wastes generated in the reconstruction of old highways is important for environmental protection and resource saving. Asphalt pavement recycling technology is used in this circumstance to solve not only the waste problem generated in the overhaul of old highways but also lengthen service life of pavements effectively [1-3]. The pavement performance is gradually degraded with time, climate changes, and increasing number of action times of traffic loads. Research on performance decay laws and establishment of decay equation for cold recycled pavement are crucial for optimizing pavement materials and structural design, improving the pavement performance, making pavement maintenance decisions and increasing returns on investments.

Compared with traditional pavements, the cold recycling technology realizes overall milling of the old pavement with a pavement milling machine, crushes and sieves recycled asphalt pavement (RAP) materials and adds a certain proportion of new aggregates and active fillers for mixing at normal temperature, followed by paving at normal temperature, to form a pavement structure layer in the end. The performance prediction and evaluation of the traditional and cold recycled pavement can be different because the cold recycling technology mainly paves the recycled mixture on the base or subbase course. Moreover, evaluation indexes of traditional asphalt pavements and their weights are inapplicable to cold recycled pavements. Project-level pavement performance prediction and evaluation can intuitively obtain the performance change curve of this project. However, the highway construction is regional, that is, transportation load, climate, and completion time vary between regions. Hence, the limited evaluation results are effective only for a single project and unsuitable for evaluating the overall level of the pavement constructed using the cold recycling technology. 
Considering the regionality of highway construction, a fuzzy prediction model and evaluation method based on road network big data were proposed to assess the performance of the cold RAP and a fuzzy prediction and evaluation system software was developed, and implemented to examine the application level of the cold recycling technology comprehensively and solve existing limitations in the project-level pavement evaluation.

\section{State of the Art}

The comprehensive performance evaluation of pavement and understanding its change laws are critical to the scientific formulation of pavement maintenance strategies. Although the evaluation of pavement performance and prediction methods have been extensively investigated [4-7], these studies are limited because they mainly take single traditional pavement engineering or single index as the evaluation object. For instance, Sabouri [8] explored the performance of asphalt mixture in the product RAP by evaluating and predicting fatigue and rutting; the results indicated that antifatigue performance can be degraded by increasing the content or RAP or reducing the content of binding agent while the rutting performance can be improved; hence, asphalt mixture with favorable economical efficiency can be produced by balancing fatigue and rutting performance. Gao et al. [9] investigated the pavement performance of top-layer and interlayer materials of the RAP, compared it with the performance of new-type asphalt mixtures with or without the same initial grading, evaluated their pavement performance, and found that the hightemperature performance of the RAP mixture reduces due to fine grading, poor low-temperature performance of the asphalt mixture, and minor influence of grading while the asphalt binder remarkably contributed to the lowtemperature performance. Suryoto et al. [10] determined functional conditions of a road using international roughness, pavement condition, and surface distress indexes and evaluated association degrees among the three indexes; the results showed that different pavement indexes caused by various function measurement methods play different roles in the selection of pavement restoration plans. Wang et al. [11] put forward an improved RAP microsurfacing mix design and material evaluation method to ensure that the performance, especially the performance of rut fillers, can satisfy field application. According to the optimized antirutting capability, optimal asphalt content (OAC) of the RAP microsurfacing mixture was determined and the effect of recycling agents on the RAP microsurfacing mixture was evaluated. The results manifested that the OAC reduces with the increase of RAP content. The recycling agent recovered initial characteristics of RAP binding agent in the microsurface layer, shortened mixing time, and improved the antirutting performance. Jing et al. [12] conducted an extensive investigation and field test of a semirigid base asphalt pavement, established a comprehensive performance evaluation system for the asphalt pavement by selecting functional and structural performance indexes, and then evaluated its comprehensive performance using principal component analysis. Sultan et al. [13] tested the RAP material, evaluated its mechanical and structural characteristics when applied to pavement construction and repair, and used the system analysis and pavement management program SAMP5 to analyze its life cycle cost by combining structural characteristics. The results indicated that improved structural characteristics of the recycled asphalt material due to the low percentage of cement allows it to be used as the stable base course for the construction and repair of new or repaired old or permanent pavement. Jamshidi et al. [14] evaluated effects of different modification methods according to material engineering characteristics and sustainability of mixed production. The results showed that the modified binding agent (output product) demonstrates the same performance level as the target binding agent. Wang et al. [15] used expressway pavement maintenance as their study object and applied a neural network to establish a performance prediction model for preventive expressway maintenance and performance evaluation. The consistent development trend results between the proposed prediction model and the measured findings indicated that the proposed model can be used to predict the pavement performance. These studies mainly concentrate on pavement material design and single performance evaluation with single evaluation objects, but investigations on the comprehensive prediction and evaluation of the pavement performance in terms of road network are lacking.

The selection of prediction and evaluation methods can exert a certain influence on the prediction and evaluation results of pavement performance. Li et al. [16] introduced the recycling of highway pavement wastes through life cycle assessment and cost analysis and analyzed the performance of the RAP from environmental and economic angles but failed to establish a multicriterion evaluation model that considers a few factors. Zhao et al. [17] used the fuzzy evaluation model to establish a quantitative evaluation system for railway tunnel diseases based on analytic hierarchy process (AHP) and established grading standards to evaluate the safety and address frequent diseases that occur in the Yiwan railway tunnel. Du [18] modeled and predicted the pavement strength, condition, and riding quality indexes using gray system theory and then compared the model with the traditional regression analysis framework. However, the overall pavement performance was difficult to evaluate because only some indexes were considered. Babashamsi et al. [19] combined the fuzzy AHP with multicriterion decision analysis while considering the pavement condition index, traffic congestion, pavement width, improvement and maintenance cost, and time needed by pavement operation to solve priority ordering problem of pavement maintenance plans. René et al. [20] predicted the development of road traffic accidents in Germany until 2015 on the basis of market diffusion of electronic stability program according to gray system theory MGM $(1,4)$. Zhang et al. [21] used the crack prediction model in pavement structure design method and the improved gray prediction model GM $(1,1)$ to predict transverse cracks of asphalt pavements in permafrost regions, discussed the complementation of the two models according to their respective advantages based on weight allocation theory, constructed a combined prediction model (PME-DGM) considering regional characteristics, and analyzed its applicability. The results showed the satisfactory effect of this method on permafrost regions in the Qinghai-Tibet Plateau. However, the proposed method only focused on permafrost regions in the Qinghai-Tibet Plateau. Abaza [22] predicted future pavement conditions on the basis of "reverse calculation" of the discrete time Markov model by using transition probability, which represents the pavement destruction rate, and then examined the influence of pavement length on the estimation of distress grade and 
transition probability. These studies evaluated single but unassociated highway pavement indexes using existing evaluation methods.

To sum up, studies on the evaluation and prediction of cold RAPs are limited. Evaluation indexes are different from indicators used in traditional pavements because the cold recycling technology mainly paves the recycled mixture on the base or subbase course. Moreover, indexes are mutually influenced; for example, pavement structure strength is correlated with pavement damage, roughness, stability, and durability. Therefore, performance indexes of the cold RAP were analyzed, cold recycled pavement evaluation indexes (CRPI) were proposed, a performance index database system based on road network big data was established for the cold RAP, and a big data-based cold recycled pavement evaluation system was set up to compensate for deficiencies in existing studies and provide a reference for understanding and evaluating performance variation trends of the road network-level cold RAP in this study.

The remainder of this study is organized as follows. Index establishment principles and evaluation and prediction methods are introduced in Section Three. Evaluation indexes are analyzed, the program setup process is introduced, and the results are provided in Section Four. Finally, the summary and related conclusions are presented in Section Five.

\section{Methodology}

\subsection{Establishment principles of the index system}

The following establishment principles of the index system and hierarchical model are presented according to characteristics of the cold RAP technology:

(1) Principle of practicability: Evaluation indexes used should practically reflect the performance and benefits of the cold RAP.

(2) Principle of hierarchy: Indexes in the same layer are compared and lower-layer indexes serve upper-layer indexes

(3) Principles of operability and feedback: Selected indexes can be effectively measured or calculated and feedback on existing problems of the project production process should be provided.

\subsection{Evaluation method}

\subsubsection{Weight determination through AHP}

The improved AHP is used to guarantee model systematicness, reasonability, and reliability through the effective combination of qualitative and quantitative analyses as well as simplify the complex calculation of the traditional AHP.

(1) Construction of judgment matrix

The judgment matrix of $A=\left(a_{i j}\right)_{n \times n}$ is expressed as follows:

$$
\begin{aligned}
& a_{i j}= \begin{cases}r_{i}-r_{j} & \left(r_{i}>r_{j}\right) \\
1 & \left(r_{i}=r_{j}\right) \\
1 /\left(r_{j}-r_{i}\right) & \left(r_{i}<r_{j}\right)\end{cases} \\
& r_{i}=\sum_{h=1}^{n} k_{i h} ; r_{j}=\sum_{h=1}^{n} k_{j h}
\end{aligned}
$$

where $a_{i j}$ is the element of the judgment matrix, $r_{i}$ and $r_{j}$ are indexes denoting the degree of importance, and $n$ is the matrix order.

$$
k_{i j}=\left\{\begin{array}{l}
0 \quad \text { i has a lower influence degree than } j \\
1 \quad \text { i has the same influence degree as } j \\
2 \quad \text { i has a higher influence degree than } j
\end{array}\right.
$$

where $i$ and $j$ are influence factors.

(2) Optimization of the judgment matrix

The original matrix is optimized to obtain the optimal matrix $B=\left(b_{i j}\right)_{n \times n}$, where

$$
b_{i j}=\sqrt[n]{\prod_{l=1}^{n} a_{l j}} / \sqrt[n]{\prod_{l=1}^{n} a_{i l}}
$$

(3) Weights of evaluation indexes

Index weights are calculated as follows:

$$
\overline{w_{i}}=\sqrt[n]{\prod_{j=1}^{n} b_{i j}}
$$

which is normalized to obtain

$$
w_{i}=\frac{\overline{w_{i}}}{\sum_{i=1}^{n} \overline{w_{i}}},(i=1,2, \cdots, n)
$$

where $w_{i}$ is the normalized weight value corresponding to the element $i$ and $n$ is the number of indexes.

\subsubsection{Determination of score threshold}

Grading standards for highway technical conditions should satisfy stipulations in Table 1 [23]. The predicted score will gradually decline with years of road operation, and the integral algorithm obtains a lower score than the grading standard because the integral evaluation follows the prediction of recycled pavement conditions and the predictive calculation is conducted in the absence of a maintenance method for the pavement.

Table 1. Subindex grading standard for pavement conditions

\begin{tabular}{l|l}
\hline Evaluation index & Score of evaluation index \\
\hline Superior & $\geqslant 90$ \\
Good & $\geqslant 80$ and $<90$ \\
Medium & $\geqslant 70$ and $<80$ \\
Inferior & $\geqslant 60$ and $<70$ \\
Poor & $<60$ \\
\hline
\end{tabular}

Hence, the grading standard of cold recycled pavement evaluation indexes and subindexes is divided into three fuzzy grades, namely, superior, medium, and poor, to consider maintenance index thresholds for highways of different levels in Shaanxi Province comprehensively [24]. The corresponding scores are listed in Table 2.

Table 2. Grading standard for evaluation indexes and subindexes of cold recycled

\begin{tabular}{l|l}
\hline Evaluation index & CRPI, PCI, RQI, RDI, and PSSI \\
\hline Superior & $\geqslant 70$ \\
Medium & $\geqslant 40$ and $<70$ \\
Poor & $<40$ \\
\hline
\end{tabular}




\subsubsection{Gray prediction model}

The gray model, a prediction method for systems containing uncertain factors, generates original data to determine system change laws, produces a data sequence with strong regularity, and establishes a corresponding differential equation model to predict future development trends of things through correlation analysis.

The gray model uses different frameworks for solving various problems. The $\operatorname{GM}(1,1)$ model is a gray model with the advantage of making accurate predictions although the original data size is small. This model is applicable to circumstances where the generated sequence presents exponential change laws and can be used to describe a monotonous change process.

$G M(1,1)$ is established as follows:

$X^{(0)}$ is set as the $\operatorname{GM}(1,1)$ modeling sequence,

$X^{(0)}=\left(x^{(0)}(1), x^{(0)}(2), \ldots, x^{(0)}(n)\right)$,

$X^{(1)}$ is (1-AGO) sequence formed through the accumulated generating operation of $X^{(0)}$.

$$
\begin{aligned}
& X^{(1)}=\left(x^{(1)}(1), x^{(1)}(2), \ldots, x^{(1)}(n)\right), \\
& x^{(1)}(t)=\sum_{i=1}^{t} x^{(0)}(i), t=1,2, \ldots, n
\end{aligned}
$$

$Z^{(1)}$ is the nearest neighbor mean (MEAN) generating sequence of $X^{(1)}$.

$$
\begin{aligned}
& Z^{(1)}=\left(z^{(1)}(1), z^{(1)}(2), \ldots, z^{(1)}(n)\right), \\
& z^{(1)}(t)=\frac{1}{2}\left(x^{(1)}(t)+z^{(1)}(t-1)\right)
\end{aligned}
$$

The $\operatorname{GM}(1,1)$ model equation is expressed as follows:

$$
\frac{d X^{(1)}(t)}{d t}+a X^{(1)}(t)=b
$$

where $\mathrm{a}$ and $\mathrm{b}$ are undetermined parameters, which are solved using the least squares method.

$$
(a, b)^{T}=\left(B^{T}, B\right)^{-1} B^{T} Y_{n}
$$

where $B=\left[\begin{array}{ll}-z^{(1)}(2) & 1 \\ -z^{(1)}(3) & 1 \\ \ldots & \ldots \\ -z^{(1)}(n) & 1\end{array}\right], \quad Y_{n}=\left[\begin{array}{l}x^{(0)}(2) \\ x^{(0)}(3) \\ \ldots \\ x^{(0)}(n)\end{array}\right]$ is:

The time response sequence of the differential equation

$$
\begin{aligned}
& \hat{X}^{(1)}(t+1)=\left[X^{(0)}(1)-\frac{b}{a}\right] e^{-a t}+\frac{b}{a} \\
& (t=1,2, \ldots, n)
\end{aligned}
$$

where $\hat{X}^{(1)}$ is the predicted value of the generated data time sequence.

The model is recovered to obtain the prediction equation as follows:

$$
\begin{aligned}
& \hat{X}^{(0)}(1)=\hat{X}^{(1)}(1)=\hat{X}^{(0)}(0) \\
& \hat{X}^{(0)}(t+1)=\hat{X}^{(1)}(t+1)-X^{(1)}(t)
\end{aligned}
$$

\subsubsection{Curve fitting through least squares method}

An approximate function is determined to fit this group of data after index values are predicted via the $\operatorname{GM}(1,1)$ model to ensure that the function approximation curve can appropriately reflect basic data trends. The least squares method is used for the curve fitting as follows:

Known data points are $\left(x_{i}, y_{i}\right), i=1,2, \ldots n$, and the fitted curve is expressed as follows:

$$
p(x)=a_{0}+a_{1}+\ldots+a_{m} x^{m}
$$

where $a_{m}$ is the coefficient and $m$ is the degree of equation.

$a_{m}$ is solved, and the matrix form of the equation set is expressed as follows:

$$
\left[\begin{array}{cccc}
1 & x_{1} & \ldots & x_{1}^{m} \\
1 & x_{2} & \ldots & x_{2}^{m} \\
\vdots & \vdots & \ddots & \vdots \\
1 & x_{n} & \ldots & x_{n}^{m}
\end{array}\right]\left[\begin{array}{c}
a_{0} \\
a_{1} \\
\vdots \\
a_{m}
\end{array}\right]=\left[\begin{array}{c}
y_{1} \\
y_{2} \\
\vdots \\
y_{n}
\end{array}\right]
$$

which is simplified to $A x=b$ and expressed as equation (16) to solve the coefficient of the fitted polynomial.

$$
\left[\begin{array}{cccc}
n & \sum x_{i} & \ldots & \sum x_{i}^{m} \\
\sum x_{i} & \sum x_{i}^{2} & \ldots & \sum x_{i}^{m+1} \\
\vdots & \vdots & \ddots & \vdots \\
\sum x_{i}^{m} & \sum x_{i}^{m+1} & \ldots & \sum x_{i}^{2 m}
\end{array}\right]\left[\begin{array}{c}
a_{0} \\
a_{1} \\
\vdots \\
a_{m}
\end{array}\right]=\left[\begin{array}{c}
\sum y_{i} \\
\sum x_{i} y_{i} \\
\vdots \\
\sum x_{i}^{n} y_{i}
\end{array}\right]
$$

3.2.5 Evaluation scores solved through definite integration

A definite integral computation is carried out for the following function after the curvilinear function $p(x)=a_{0}+a_{1} x+\ldots+a_{m} x^{m}$ is fitted using subindex values in each phase:

$I=\int_{1}^{b} p(x) d x$

where $I$ is the total score of subindexes and $\mathrm{b}$ is the number of pavement test times.

The final score is:

$$
S=\frac{I}{b}
$$

\section{Result Analysis and Discussion}

\subsection{C\# introduction}

$\mathrm{C \#}$ is a safe, stable, simple, and element object-oriented programming language derived from $\mathrm{C}$ and $\mathrm{C}++$. $\mathrm{CH}$ excludes some complex characteristics of $\mathrm{C}$ and $\mathrm{C}++$ while optimizing their powerful functions. This programming language has been widely used in .NET development due to its powerful operability, elegant grammar style, innovative language features, and convenient module programmingoriented support by integrating the simple visualized operation of VB and high operation efficiency of $\mathrm{C}++$. 
A database management system of Shaanxi cold recycled pavement was established in this study using statistics of index data of technical conditions for cold RAPs in Shaanxi Province. Cold recycling technology projects were classified according to intervals of years of their operation after completion, and subindex data on cold recycling technical conditions were calculated by category. All the life-cycle change trends of subindexes were predicted according to the statistical information of the powerful database, and cold recycling technical conditions can be comprehensively evaluated according to the integral of the change curve.

\subsection{Index analysis}

A certain proportion of new materials, such as new aggregate, recycling binder, and asphalt recycling agent, are mixed with materials recycled from asphalt pavement, including recycled asphalt mixture and a certain proportion of inorganic recycled materials, in the asphalt pavement recycling process. These materials are paved and compacted to form a pavement structure layer. The cold recycling technology mainly paves the recycled mixture on the base or subbase course. Therefore, selected evaluation indexes should be able to reflect the performance of the pavement base course and the overall structural performance.

CRPI, pavement surface condition (PCI), riding quality (RQI), rutting depth (RDI), and pavement structure strength (PSSI) indexes are proposed in this study.

\subsubsection{Pavement surface condition index}

PCI comprehensively characterizes the pavement damage on the basis of the pavement damage rate. This index is a comprehensive reflection of pavement diseases with varying degrees and pavement performance. Pavement diseases, such as cracks and pit slots, are correlated with the base course of the pavement when the recycled mixture serves as the base course.

\subsubsection{Riding quality index}

Road surface roughness refers to the deviation value of longitudinal concave-convex amounts on the road surface and is evaluated using RQI. As an important index in pavement evaluation and delivery-receiving acceptance, RQI mainly reflects the roughness of the profile curve of the pavement's vertical section. Although the cold recycling technology mainly paves the mixture on the base or subbase course, the roughness or concave-convex subsidence of the base or subbase course will seriously impact the overall road surface roughness.

\subsubsection{Rutting depth index}

Rut is a permanent wheel indentation that remains on the pavement where vehicles run. The rutting depth directly reflects the vehicle running comfort as well as the safety and service life of the pavement. The pavement performance and quality can be intuitively reflected through the rutting depth test and evaluation.

\subsubsection{Pavement structure strength index}

Pavement structure strength is evaluated via PSSI. The pavement structure performance is the degree to which a pavement structure resists external load and environmental factors and maintains its intact condition, and it is mainly reflected by its load-carrying capacity. The load-carrying capacity of the pavement structure is the basis for its service performance that presents internal relations with pavement damage, roughness, stability, and durability. The load- bearing capacity of the pavement structure will gradually deteriorate during the service period while various types of damage evolve in each step. The damage development speed of road segment with strong load-carrying capacity is commonly low, while the road segment with weak loadcarrying capacity demonstrates a rapid damage development speed. Therefore, determining change laws of pavement structure strength is crucial in the correct evaluation of pavement quality and scientific formulation of road maintenance strategies.

CRPI is calculated as follows:

$$
C R P I=w_{P C I} P C I+w_{R Q I} R Q I+w_{R D I} R D I+w_{P S S I} P S S I
$$

where $w$ is the index weight.

\subsection{Database design}

Database is a warehouse that organizes, saves, and manages data according to their structure. Access database management system is used in this study, and several datasheets are established to save different types of information. The name, code, and type of data items are listed in Table 3.

Table 3. Datasheet information

\begin{tabular}{|c|c|c|}
\hline Field name & Data type & Description \\
\hline Id & $\begin{array}{c}\text { Auto } \\
\text { numbering }\end{array}$ & Auto numbering \\
\hline ProName & Short text & Project name \\
\hline ProYear & Number & Years of operation upon test \\
\hline PCI & Number & Pavement surface condition index \\
\hline RDI & Number & Rutting depth index \\
\hline RQI & Number & Riding quality index \\
\hline PSSI & Number & Pavement structure strength index \\
\hline
\end{tabular}

\subsection{Program setup}

The cold recycled pavement prediction and evaluation system integrates information entry, data query, computational analysis, and result display. Data are classified and summarized according to data type and number of test times of each pavement index after the cold recycled pavement predictive index database, and codes are fitted using the gray prediction model and least squares method to realize comprehensive prediction and evaluation. This program is mainly divided into three modules, namely, information entry, database operation, statistics and calculation, and result display. The program flowchart is shown in Fig. 1.

\section{(1) Information addition module}

Project names, years of pavement operation (upon test), and index values should be inputted when the project information is added in the system. Classifying the input information of the cold recycled pavement according to years of highway operation upon the index test is necessary because this big data-based database evaluation system used to evaluate the performance of cold recycled pavements ignores the number of years highways have been constructed. Hence, performance indexes of the cold recycled pavement should be summarized, the development of pavement performance must be predicted according to annual test values of pavement performance indexes, and the pavement performance should be comprehensively evaluated. The interface of information addition module is shown in Fig. 3. 


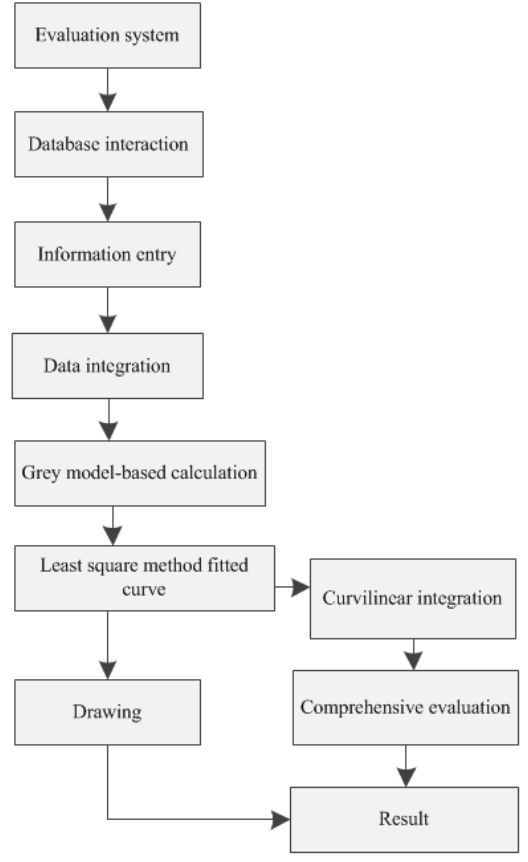

Fig. 1. Program flowchart

The evaluation software program based on $\mathrm{C} \#$ includes four modules, as shown in Fig. 2.

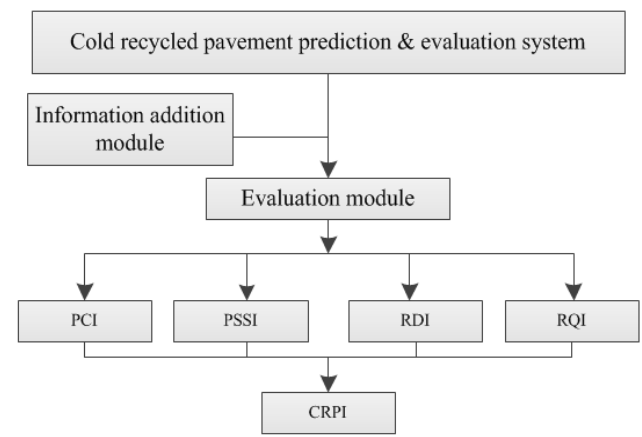

Fig. 2. Software modules

Evaluation System of Cold R... $\quad-\quad \square \quad \times$ A.dd... PCI RDI RQI PSSI CRPI

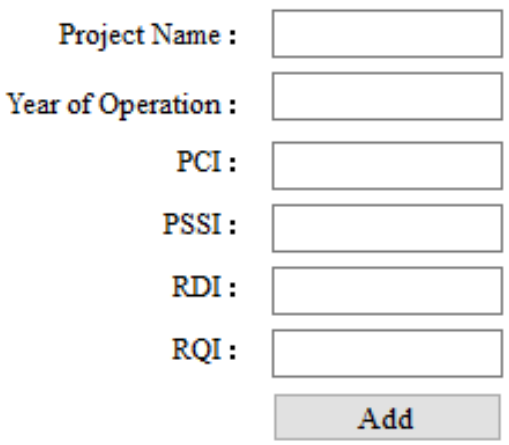

Fig. 3. Interface of information addition

(2) Evaluation module

The evaluation module is divided into evaluation views of five indexes, namely, CRPI, PCI, RQI, RDI, and PSSI. Each index includes the predicted value, relative error, prediction curve chart, and index score. An example of the interface of the evaluation module is illustrated in Fig. 4.

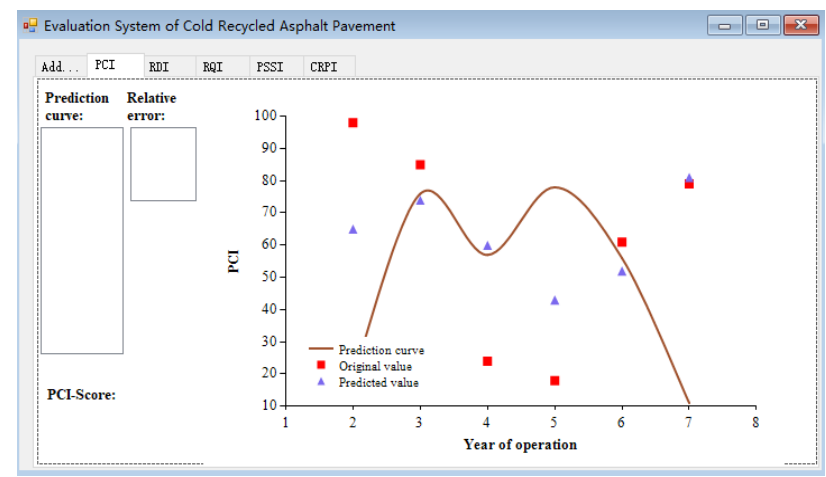

Fig. 4. Interface of evaluation module (example)

\subsection{The Program application}

Test data after the highway operates for a certain number of years should exist in the database and be classified according to years of operation and the performance index value in each year must be the mean value of all indexes in this year before the cold recycled base course pavement is evaluated. The mean value of indexes is first calculated according to years of operation; the future service life index of the pavement is calculated using the gray prediction model (Formulas (70)-(13)); the performance development trend of the pavement is examined via fitted curves of the least squares method (Formulas (140-(16)), followed by the integral operation of the prediction curve; the mean value is solved (Formulas (17)-(18)), and the overall score of each single index is finally obtained during the programmed computation.

\subsubsection{Determination of index weights}

The final CRPI value is calculated using Formula (19) after the score of each single index is solved. Index weights are calculated via the improved AHP (Formulas (1)-(6)) and listed in Table 4.

Table. 4. Index weights

\begin{tabular}{l|l|l|l|l}
\hline Subindex & PCI & RQI & RDI & PSSI \\
\hline Weight $(w)$ & 0.20 & 0.07 & 0.20 & 0.53 \\
\hline
\end{tabular}

Table 4 shows that the weight of PSSI is the maximum in the pavement evaluation because the load-carrying capacity of the pavement structure is the basis for its service performance that presents internal relations with pavement damage, roughness, stability, and durability. Given that cold recycling of old materials is mainly applied to pavement base and subbase courses, it has a minor effect on the pavement roughness. Hence, the weight of RQI is the minimum.

\subsubsection{Prediction and evaluation results}

The evaluation program is based on big data statistics of cold recycled base course pavements. The high input of scores of pavement performance indexes enhances the representativeness of scores. Only some test data of the pavement performance are taken as examples in this study to test the system and realize the performance evaluation and analysis. Original test values of pavement indexes are listed in Table 5.

\begin{tabular}{l} 
Table. 5. Original test values of indexes \\
\hline \multirow{2}{*}{ Number of test times } \\
\hline \multirow{2}{*}{1}
\end{tabular}




\begin{tabular}{l|l|l|l|l}
\hline \multirow{2}{*}{2} & 95.2 & 95.3 & 90 & 96.16 \\
& 95.0 & 94.7 & 86.2 & 95.88 \\
\hline \multirow{2}{*}{3} & 94.1 & 94.6 & 86.2 & 94.62 \\
& 94.8 & 93.2 & 85.3 & 95.60 \\
\hline \multirow{2}{*}{4} & 91.2 & 91.0 & 82.4 & 90.57 \\
& 93.3 & 90.7 & 82.9 & 93.50 \\
\hline
\end{tabular}

Original test values of indexes are inputted into the database from the program's information addition interface. The final evaluation results are presented in Figs. 5-9.

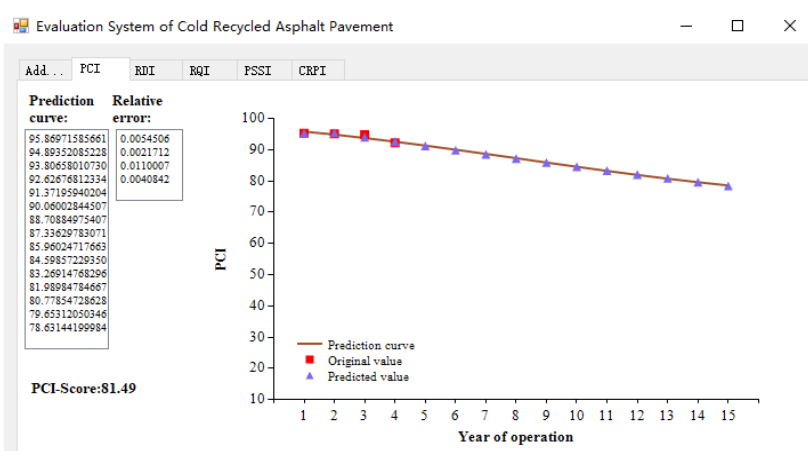

Fig. 5. PCI evaluation curve

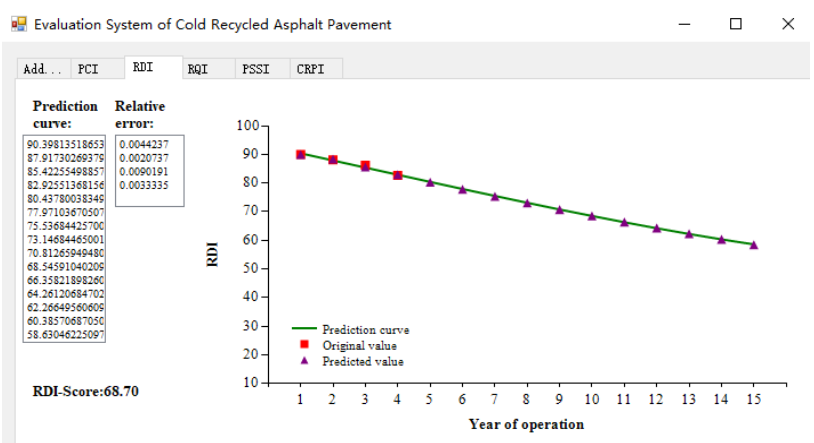

Fig. 6. RDI evaluation curve

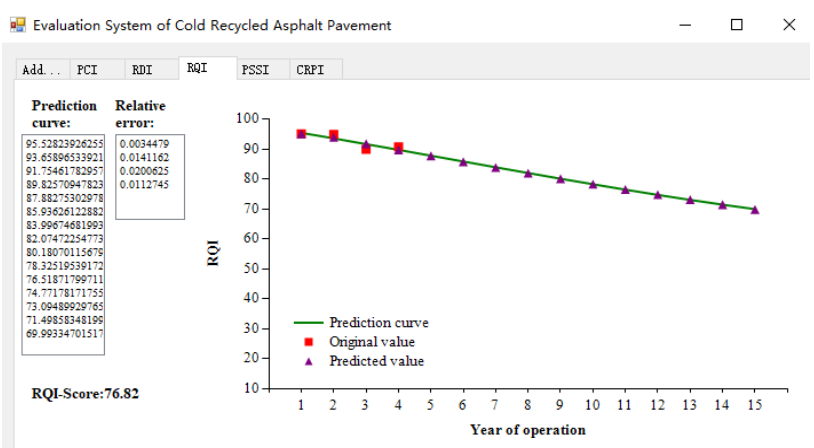

Fig. 7. RQI evaluation curve

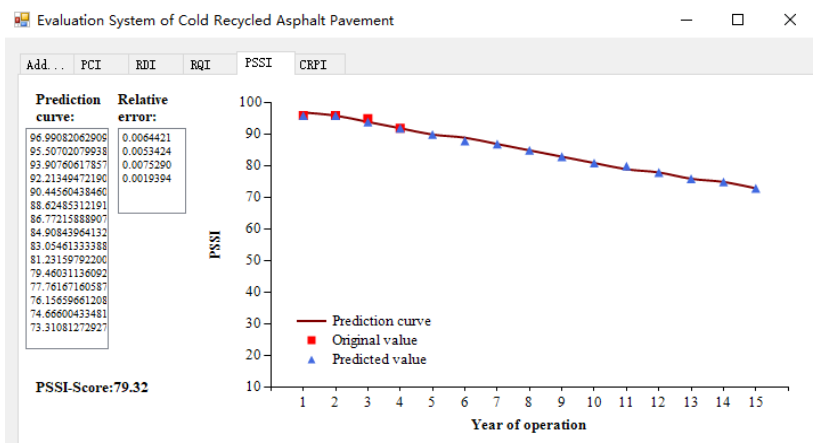

Fig. 8. PSSI evaluation curve

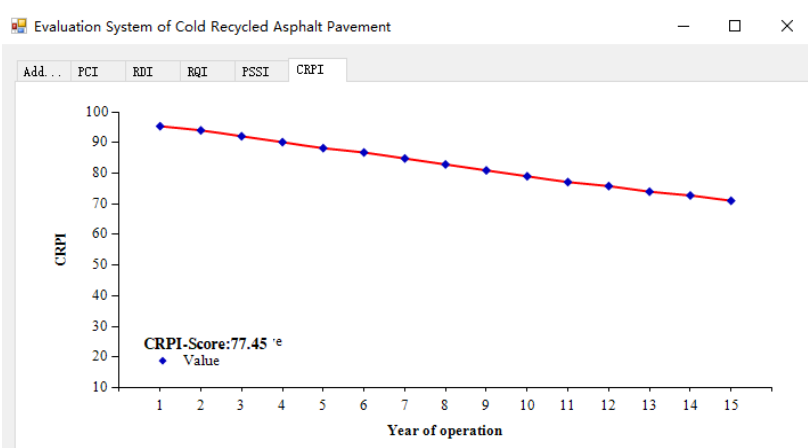

Fig. 9. CRPI evaluation curve

The computational analysis of data inputted in the database shows that the CRPI value is 77.45 (Figs. 5-9). Table 3-2 demonstrates that the pavement is superior based on the grading standard for pavement condition subindexes. Subindexes of the pavement in this region exhibit good performance because PCI, RDI, RQI, and PSSI scores are $81.49,68.70,76.82$, and 79.32 , respectively. The prediction curve fits appropriately with original data with small relative errors. The analysis of various index evaluation curves shows the gradual overall downtrend of performance indexes tends and generally acceptable pavement performance.

\section{Conclusions}

The software program was compiled and the gray prediction model was combined with the improved AHP to analyze and discuss the performance evaluation of the cold RAP as well as establish a fuzzy prediction and evaluation system for assessing the performance of cold RAP. The following conclusions were mainly drawn from this study:

(1) Evaluation indexes and subindexes based on big data statistics were proposed for the cold recycled pavement by combining cold recycling technical features. Hierarchical and operable evaluation indexes reflected the performance of the pavement base course and overall structural performance and provided feedback.

(2) The improved AHP was used to determine weights of evaluation indexes for the cold recycled pavement. The results showed that the weight of PSSI was the maximum. Thresholds of major and medium repair and maintenance of the asphalt pavement were combined to determine fuzzy grading standards for evaluation indexes and subindexes. Evaluation grades were divided into three standards, namely, superior, medium, and poor.

(3) The GM $(1,1)$ model was adopted to evaluate the current status of the cold recycled pavement and predict the future pavement condition index. The curve of prediction results was fitted to obtain the predictor formula of the pavement condition, followed by definite integral computation of the predictor formula to finally calculate the comprehensive score.

(4) A big data-based cold recycled pavement evaluation system containing modules, such as data entry and result analysis, was set up. The results showed that evaluation indexes of the cold recycled pavement can be predicted on the basis of gray prediction theory and high prediction accuracy can be realized. This system can realize the programming and simplification of the statistical analysis and evaluation work for the cold recycled pavement condition. 
Hence, the big data-based fuzzy prediction and evaluation system proposed in this study can be used to predict the performance of road network-level cold RAP and its performance development trend. The results of this study can provide a theoretical foundation for evaluating and analyzing the performance of cold recycled pavements. However, the score interval of the evaluation grade is large and the final evaluation result is fuzzy because the pavement evaluation criteria vary for each pavement at different levels. Therefore, the investigation and analysis of the cold recycling technology should be strengthened and evaluation grading standards must be improved to enhance the fuzziness of the evaluation results and contribute to the big data-based performance evaluation of cold recycled pavements in the future.

This is an Open Access article distributed under the terms of the Creative Commons Attribution License.

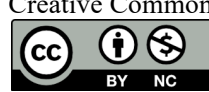

\section{References}

1. Gu, F., Ma, W. Y., West, R. C., Taylor, A. J., Zhang, Y. Q., "Structural performance and sustainability assessment of cold central-plant and in-place recycled asphalt pavements: A case study". Journal of Cleaner Production, 208, 2018, pp.1513-1523.

2. Ji, S. Y., "Study on Application of Emulsified Asphalt Cold Regeneration Base". Master thesis of Harbin Institute of Technology, China, 2018, pp.1-13.

3. Yu, Y. T., "Research on Cold Recycling Technology with Foamed Bitumen Based on Cement Stabilization Mixing Plant". Master thesis of South China University of Technology, China, 2018, pp.113.

4. Mandolini, M., Favi, C., Germani, M., "An Analytic Hierarchy Method For Anticipating The Selection Of The Supplying Strategy At The Design Stage". Dyna, 94(2), 2019, pp.161-167.

5. Solonenko, I., "The use of cement concrete pavements for roads, depending on climatic conditions". Tehnički glasnik, 13(3), 2019, pp.235-240.

6. Tao, J., Fu, Z. W., Gao, H., "Air Bending of Sheet Metal Based on the Grey Prediction Model”. Journal of Engineering Science and Technology Review, 12(3), 2019, pp.123-129.

7. Farjah, E., "Proposing an Efficient Wind Forecasting Agent Using Adaptive MFDFA". Journal of Power Technologies, 99(2), 2019, pp.152-162.

8. Sabouri, M., "Evaluation of performance-based mix design for asphalt mixtures containing Reclaimed Asphalt Pavement (RAP)". Construction and Building Materials, 235, 2020, pp.117545.

9. Gao, L., Li, L., Xie, J. H., Yu, Z. G., Charmot, S., "Evaluation of pavement performance for reclaimed asphalt materials in different layers". Construction and Building Materials, 159, 2018, pp.561566.

10. Suryoto, Siswoyo, D. P., Setyawan, A., "The Evaluation of Functional Performance of National Roadway using Three Types of Pavement Assessments Methods". Procedia Engineering, 171, 2017, pp.1435-1442.

11. Wang, A. P., Shen, S. H., Li, X. H., Song, B., "Micro-surfacing mixtures with reclaimed asphalt pavement: Mix design and performance evaluation". Construction and Building Materials, 201, 2019, pp.303-313.

12. Jing, C., Zhang, J. X., Song, B., “An innovative evaluation method for performance of in-service asphalt pavement with semi-rigid base". Construction and Building Materials, 235, 2020, pp.117376.

13. Sultan, S. A., Guo, Z. Y., "Evaluating the Performance of Sustainable Perpetual Pavements using Recycled Asphalt Pavement in China". International Journal of Transportation ence \& Technology, 5(3), 2016, pp.200-209.
14. Jamshidi, A., Hamzah, M. O., Kurumisawa, K., Nawa, T., Samali, B., "Evaluation of sustainable technologies that upgrade the binder performance grade in asphalt pavement construction". Materials \& Design, 95, 2016, pp.9-20.

15. Wang, Z. C., Guo, N. S., Wang, S., Xu, Y., "Prediction of highway asphalt pavement performance based on Markov chain and artificial neural network approach". The Journal of Supercomputing, 77(2), 2020. pp.1354-1376.

16. Li, J., Xiao, F. P., Zhang, L. F., Amirkhanian, S. N., "Life cycle assessment and life cycle cost analysis of recycled solid waste materials in highway pavement: A review". Journal of Cleaner Production, 233, 2019, pp.1182-1206.

17. Zhao, B. J., Kong, D. K., Tan, Z. S., "Yichang-Wanzhou railway tunnel disease evaluation system based on analytic hierarchy process". China Civil Engineering Journal, 50(S2), 2017, pp.243248 .

18. Du, E. P., Ma, S. L., Jing, H. M., “Asphalt Pavement Performance Prediction Model Based on Gray System Theory". Journal of TongJi University (Natural science), 38(8), 2010, pp.1161-1164.

19. Babashamsi, P., Golzadfar, A., Yusoff, N. I. M., Ceylan, H., Nor, N. G. M., "Integrated fuzzy analytic hierarchy process and VIKOR method in the prioritization of pavement maintenance activities". International Journal of Pavement Research and Technology, 9(2), 2016, pp.112-120.

20. René S, H., Becker, U., Manz, H., "Grey systems theory time series prediction applied to road traffic safety in Germany". IFACPapersOnLine, 49(3), 2016, pp.231-236.

21. Zhang, C., Wang, H. N., Yang, X., You, Z. P., "A Combinational Prediction Model for Transverse Crack of Asphalt Pavement". KSCE Journal of Civil Engineering, 22(6), 2018, pp.2109-2117.

22. Abaza, K. A., "Back-calculation of transition probabilities for Markovian-based pavement performance prediction models". International Journal of Pavement Engineering, 17(3), 2016, pp.253-264.

23. Industry Standards of the People's Republic of China, "Highway performance assessment standards: JTG 5210-2018". Beijing: Ministry of Transport of the People's Republic of China Press, China, 2018, pp.17-21.

24. Huo, Q., "Research on maintenance indices' threshold of Shaanxi common trunk highway asphalt pavement". Master thesis of Chang'an University, China, 2017, pp.62-67. 